In a special issue in memory of Don Carlson in Journal of Elasticity

\title{
Secular Equations for Rayleigh and Stoneley Waves in Exponentially Graded Elastic Materials of General Anisotropy under the Influence of Gravity
}

T. C. T. Ting ${ }^{*} 1$

This paper is dedicated to, and in memory of, Professor Donald E. Carlson.

\begin{abstract}
The Stroh formalism is employed to study Rayleigh and Stoneley waves in exponentially graded elastic materials of general anisotropy under the influence of gravity. The $6 \times 6$ fundamental matrix $\mathbf{N}$ is no longer real. Nevertheless the coefficients of the sextic equation for the Stroh eigenvalue $p$ are real. The orthogonality and closure relations are derived. Also derived are three Barnett-Lothe tensors. They are not necessarily real. Secular equations for Rayleigh and Stoneley wave speeds are presented. Explicit secular equations are obtained when the materials are orthotropic. In the literature, the secular equations for Stoneley waves in orthotropic materials are obtained without using the Stroh formalism. As a result, it requires computation of a $4 \times 4$ determinant. The secular equation presented here requires computation of a $2 \times 2$ determinant, and hence is fully explicit. A Rayleigh or Stoneley wave exists in the exponentially graded material under the influence of gravity if the wave can propagate in the homogeneous material without the influence of gravity. As the wave number $k \rightarrow \infty$, the Rayleigh or Stoneley wave speed approaches the speed for the homogeneous material.
\end{abstract}

Key words: anisotropic; inhomogeneous materials; exponentially graded materials; secular equations; Rayleigh waves; Stoneley waves; Stroh formalism; influence of gravity.

Mathematical Subject Classifications: 74B05, 74J05, 74J15

\footnotetext{
* Professor Emeritus of University of Illinois at Chicago

${ }^{1}$ Division of Mechanics and Computation, Stanford University, Durand 262, Stanford, CA 94305, USA e-mail: tting@uic.edu
} 


\section{Introduction}

Surface waves in elastic half-space $x_{2} \geq 0$ in which the material property depends on the depth $x_{2}$ have been of interest in recent years [1-12]. Some of them studied anti-plane shear surface waves that are possible only for certain special anisotropic elastic materials. For a general graded material, anti-plane shear surface waves for large wave number were considered by Achenbach and Balogun [11] for isotropic elastic materials and by Ting [12] for anisotropic elastic materials.

Rayleigh type surface waves in exponentially graded orthotropic materials were investigated by Destrade [3]. He showed that the quartic equation for the Stroh eigenvalue $p$ is, after properly modified, a quadratic equation in $p^{2}$ with real coefficients. He also showed that the displacement and the stress decay at different rates with the depth $x_{2}$ of the half-space. Vinh and Seriani [7] studied the same problem and added the influence of gravity on surface waves.

There were several studies on the influence of gravity on Rayleigh waves [7, 13-14], Lamb waves [15] and Stoneley waves [16, 17]. Only special anisotropic materials such as orthotropic materials have been considered. Rayleigh waves in exponentially graded elastic materials of general anisotropy under the influence of gravity were investigated in [18].

In this paper we study Rayleigh surface waves and Stoneley waves in exponentially graded anisotropic elastic materials under the influence of gravity. The influence of gravity is an important factor in studying surface waves on the earth generated by an earthquake. Since the earth need not be orthotropic, we consider the materials of general anisotropy. Basic equations for surface waves in an exponentially graded elastic material of general anisotropy under the influence of gravity are presented in Section 2 using the Stroh's formalism [19-21]. The $6 \times 6$ fundamental matrix $\mathbf{N}$ is no longer real. The imaginary parts come from the inhomogeneity of the material and the influence of gravity. General solutions for the displacement and the stress function are presented and the Stroh eigenrelation for the eigenvalue $p$ is obtained. In Section 3 we show that the coefficients of the sextic equation for $p$ are real even though the matrix $\mathbf{N}$ is complex. The orthogonality relations of the right and left eigenvectors are established and the closure 
relations are obtained from which we re-define the three Barnett-Lothe tensors. They are not necessarily real. Rayleigh surface waves are considered in Section 4. With the three Barnett-Lothe tensors, the secular equation for the surface wave speed can have several different forms. For the special case of orthotropic materials, explicit secular equation for the surface wave speed is obtained. Stoneley waves are investigated in Section 5. Secular equation for the Stoneley wave speed is presented. Again, explicit secular equation for the Stoneley wave speed is obtained when the materials are orthotropic. Since the analysis recover the case of homogeneous materials without the influence of gravity when the wave number $k$ is very large, if a surface wave or a Stoneley wave exists for homogeneous materials without the influence of gravity, it exists also for the exponentially graded elastic materials of general anisotropy under the influence of gravity when the wave number $k$ is very large. As $k \rightarrow \infty$, the wave speed for the exponentially graded elastic materials of general anisotropy under the influence of gravity approaches the wave speed for the homogeneous materials without the influence of gravity.

In the paper, all equations are derived in such a way that, when the material is homogeneous without the influence of gravity, they recover the known equations in the literature.

\section{The Stroh formalism}

In a fixed rectangular coordinate system $x_{i} \quad(i=1,2,3)$, let $g$ be the gravitational acceleration that is in the direction of the $x_{2}$-axis. The equation of motion can be written as $[16,22]$

$$
\begin{gathered}
\sigma_{11,1}+\sigma_{12,2}+\sigma_{13,3}+\hat{\rho} g u_{2,1}=\hat{\rho} w_{1}^{\prime \prime}, \\
\sigma_{21,1}+\sigma_{22,2}+\sigma_{23,3}-\hat{\rho} g\left(u_{1,1}+u_{3,3}\right)=\hat{\rho} \mathbb{W}_{2}, \\
\sigma_{31,1}+\sigma_{32,2}+\sigma_{33,3}+\hat{\rho} g u_{2,3}=\hat{\rho} \text { W, }
\end{gathered}
$$

where $\sigma_{i j}$ is the stress, $u_{i}$ is the displacement, $\hat{\rho}$ is mass density which depends on $x_{2}$, the dot denotes differentiation with time $t$ and a comma denotes differentiation with $x_{i}$. The sign convention for $g$ here follows [16, 22]. The sign convention in [13, 14] employed the opposite direction for the gravitational acceleration so that the $g$ here is $-g$ 
in $[13,14]$. For the problem to be studied here, $\sigma_{i j}$ and $u_{i}$ do not depend on $x_{3}$ so that (2.1) can be written as

$$
\sigma_{i 1,1}+\sigma_{i 2,2}+\hat{\rho} g Y_{i k} u_{k, 1}=\hat{\rho} w_{i}^{\prime}, \quad(i=1,2,3),
$$

where $\mathbf{Y}$ is an anti-symmetric matrix

$$
\mathbf{Y}=\left\lfloor\begin{array}{ccc}
0 & 1 & 0 \\
-1 & 0 & 0 \\
0 & 0 & 0
\end{array}\right\rfloor
$$

Consider a steady wave propagating in the direction of the $x_{1}$-axis with wave speed $v$ so that

$$
\mathbf{u}=\mathbf{u}\left(x_{1}-v t, x_{2}\right)
$$

This means that

$$
\ddot{\mathbf{W}}=v^{2} \mathbf{u}, 11 \text {. }
$$

The equation of motion (2.2) can then be written as

$$
\left(\sigma_{i 1}+\hat{\rho} g Y_{i k} u_{k}-\hat{\rho} v^{2} u_{i, 1}\right)_{, 1}+\left(\sigma_{i 2}\right)_{, 2}=0
$$

assuming that $\hat{\rho}$ is independent of $x_{1}$. Equation (2.6) tells us that there exists a stress function $\phi$ such that [20]

$$
\sigma_{i 1}+\hat{\rho} g Y_{i k} u_{k}-\hat{\rho} v^{2} u_{i, 1}=-\phi_{i, 2}, \quad \sigma_{i 2}=\phi_{i, 1} \text {. }
$$

The stress-strain relation is

$$
\begin{gathered}
\sigma_{i j}=\hat{C}_{i j k s} u_{k, s}, \\
\hat{C}_{i j k s}=\hat{C}_{j i k s}=\hat{C}_{k s i j}=\hat{C}_{i j s k},
\end{gathered}
$$

in which $\hat{C}_{i j k s}$ is the elastic stiffness that depends on $x_{2}$. The $\hat{C}_{i j k s}$ is positive definite and possesses the full symmetry shown in (2.9). The third equality in (2.9) is redundant because the first two imply the third ([21], p.32). From (2.8) we have

$$
\begin{gathered}
\sigma_{i 1}=\hat{Q}_{i k} u_{k, 1}+\hat{R}_{i k} u_{k, 2}, \\
\sigma_{i 2}=\hat{R}_{k i} u_{k, 1}+\hat{T}_{i k} u_{k, 2},
\end{gathered}
$$

where

$$
\hat{Q}_{i k}=\hat{C}_{i 1 k 1}, \quad \hat{R}_{i k}=\hat{C}_{i 1 k 2}, \quad \hat{T}_{i k}=\hat{C}_{i 2 k 2}
$$


The matrices $\hat{\mathbf{Q}}$ and $\hat{\mathbf{T}}$ are symmetric and positive definite. Substitution of (2.10) into (2.7) leads to

$$
\left[\begin{array}{cc}
\hat{\boldsymbol{\rho}} v^{2} \mathbf{I}-\hat{\mathbf{Q}} & \mathbf{0} \\
-\hat{\mathbf{R}}^{T} & \mathbf{I}
\end{array}\right]\left[\begin{array}{l}
\mathbf{u}, 1 \\
\boldsymbol{\phi}, 1
\end{array}\right]-\hat{\rho} g\left[\begin{array}{ll}
\mathbf{Y} & \mathbf{0} \\
\mathbf{0} & \mathbf{0}
\end{array}\right]\left[\begin{array}{l}
\mathbf{u} \\
\boldsymbol{\phi}
\end{array}\right]=\left[\begin{array}{cc}
\hat{\mathbf{R}} & \mathbf{I} \\
\hat{\mathbf{T}} & \mathbf{0}
\end{array}\right]\left[\begin{array}{l}
\mathbf{u}, 2 \\
\boldsymbol{\phi}, 2
\end{array}\right] .
$$

If we multiply both sides by the matrix

$$
\left\lfloor\begin{array}{cc}
\mathbf{0} & \hat{\mathbf{T}}^{-1} \\
\mathbf{I} & -\hat{\mathbf{R}} \hat{\mathbf{T}}^{-1}
\end{array}\right\rfloor
$$

we have

$$
\left[\begin{array}{cc}
\hat{\mathbf{N}}_{1} & \hat{\mathbf{N}}_{2} \\
\hat{\mathbf{N}}_{3}+\hat{\rho} v^{2} \mathbf{I} & \hat{\mathbf{N}}_{1}^{T}
\end{array}\right]\left[\begin{array}{l}
\mathbf{u}, 1 \\
\phi, 1
\end{array}\right]-\hat{\rho} g\left[\begin{array}{ll}
\mathbf{0} & \mathbf{0} \\
\mathbf{Y} & \mathbf{0}
\end{array}\right]\left[\begin{array}{l}
\mathbf{u} \\
\phi
\end{array}\right]=\left[\begin{array}{l}
\mathbf{u}, 2 \\
\phi, 2
\end{array}\right]
$$

where [23]

$$
\hat{\mathbf{N}}_{1}=-\hat{\mathbf{T}}^{-1} \hat{\mathbf{R}}^{T}, \quad \hat{\mathbf{N}}_{2}=\hat{\mathbf{T}}^{-1}, \quad \hat{\mathbf{N}}_{3}=\hat{\mathbf{R}} \hat{\mathbf{T}}^{-1} \hat{\mathbf{R}}^{T}-\hat{\mathbf{Q}} .
$$

$\hat{\mathbf{N}}_{2}$ is symmetric and positive definite while $-\hat{\mathbf{N}}_{3}$ is symmetric and positive semi-definite. Equation (2.13) is a matrix differential equation for the displacement $\mathbf{u}$ and the stress function $\phi$. The elastic stiffness $\hat{C}_{i j k s}$ and the mass density $\hat{\rho}$ can depend on $x_{2}$ arbitrarily. For elastostatics for which $v=0, \hat{C}_{i j k s}$ can depend on $x_{1}$ also ([21], p.150). If $g=0, \hat{\rho}$ can also depend on $x_{1}$.

We assume that the elastic stiffness $\hat{C}_{i j k s}$ and the mass density $\hat{\rho}$ depend on $x_{2}$ exponentially as

$$
\hat{C}_{i j k s}=C_{i j k s} e^{-2 \alpha x_{2}}, \quad \hat{\rho}=\rho e^{-2 \alpha x_{2}},
$$

where $C_{i j k s}, \rho$ and $\alpha$ are constants. The exponential factor $\alpha$ can be positive or negative. Equation (2.13) reduces to

$$
\left[\begin{array}{cc}
\mathbf{N}_{1} & e^{2 \alpha x_{2}} \mathbf{N}_{2} \\
e^{-2 \alpha x_{2}}\left(\mathbf{N}_{3}+\rho v^{2} \mathbf{I}\right) & \mathbf{N}_{1}^{T}
\end{array}\right]\left[\begin{array}{l}
\mathbf{u}_{1} \\
\phi_{, 1}
\end{array}\right]-\rho g e^{-2 \alpha x_{2}}\left[\begin{array}{ll}
\mathbf{0} & \mathbf{0} \\
\mathbf{Y} & \mathbf{0}
\end{array}\right]\left\lfloor\begin{array}{l}
\mathbf{u} \\
\phi
\end{array}\right]=\left\lfloor\begin{array}{l}
\mathbf{u}, 2 \\
\phi_{, 2}
\end{array}\right\rfloor,
$$

where $\mathbf{N}_{1}, \mathbf{N}_{2}$ and $\mathbf{N}_{3}$ are constants. Let

$$
\phi=e^{-2 \alpha x_{2}} \boldsymbol{\Phi} .
$$


Equation (2.16) simplifies to

$$
\left[\begin{array}{cc}
\mathbf{N}_{1} & \mathbf{N}_{2} \\
\mathbf{N}_{3}+\rho v^{2} \mathbf{I} & \mathbf{N}_{1}^{T}
\end{array}\right]\left[\begin{array}{l}
\mathbf{u}_{1} \\
\boldsymbol{\Phi}, 1
\end{array}\right]-\rho g\left[\begin{array}{cc}
\mathbf{0} & \mathbf{0} \\
\mathbf{Y} & \mathbf{0}
\end{array}\right]\left[\begin{array}{l}
\mathbf{u} \\
\boldsymbol{\Phi}
\end{array}\right]=\left[\begin{array}{c}
\mathbf{u}_{2} \\
\boldsymbol{\Phi}, 2-2 \alpha \boldsymbol{\Phi}
\end{array}\right],
$$

which is a system of partial differential equations in $\mathbf{u}$ and $\Phi$ with constant coefficients. A general solution is

$$
\begin{gathered}
\mathbf{u}=\mathbf{a} e^{i k\left[x_{1}-v t+(p-i \beta) x_{2}\right]}, \\
\Phi=\mathbf{b} e^{i k\left[x_{1}-v t+(p-i \beta) x_{2}\right]},
\end{gathered}
$$

in which $p$, $\mathbf{a}$ and $\mathbf{b}$ are constant, $k$ is the real wave number and

$$
\beta=\alpha / k \text {. }
$$

Substitution of (2.19) into (2.18) leads to

$$
\begin{gathered}
\mathbf{N} \xi=p \xi \\
\mathbf{N}=\left[\begin{array}{cc}
\mathbf{N}_{1}+i \beta \mathbf{I} & \mathbf{N}_{2} \\
\mathbf{N}_{3}+X \mathbf{I}+i h \mathbf{Y} & \mathbf{N}_{1}^{T}-i \beta \mathbf{I}
\end{array}\right], \xi=\left[\begin{array}{l}
\mathbf{a} \\
\mathbf{b}
\end{array}\right]
\end{gathered}
$$

In the above,

$$
X=\rho v^{2}, \quad h=\rho g / k .
$$

When $\beta=0$ and $h=0,(2.21)$ recovers the Stroh eigenrelation for homogeneous materials without the gravity. However, unless $\beta$ and $h$ both vanish, the $6 \times 6$ matrix $\mathbf{N}$ is complex.

From $(2.17)$ and $(2.19)_{2}$, the stress function is

$$
\phi=\mathbf{b} e^{i k\left[x_{1}-v t+(p+i \beta) x_{2}\right]}
$$

Equations (2.19) $)_{1}$ and (2.24) tell us that the displacement and stress decay exponentially at different rates with the factors $k[\operatorname{Im}(p)-\beta]$ and $k[\operatorname{Im}(p)+\beta]$, respectively. This was first discovered by Destrade [3] for orthotropic materials. For the displacement and stress to vanish at $x_{2}=\infty$ we must have

$$
\operatorname{Im}(p)>|\beta|
$$

It is not difficult to show that exponentially graded materials are the only ones for which the matrix differential equation (2.13) has a closed form solution. 


\section{The Barnett-Lothe tensors}

Despite the fact that the $6 \times 6$ matrix $\mathbf{N}$ is complex, it is shown in [18] that the coefficients of the sextic equation in $p$ are real. Introducing the $6 \times 6$ symmetric matrix

$$
\mathbf{J}=\left\lfloor\begin{array}{ll}
\mathbf{0} & \mathbf{I} \\
\mathbf{I} & \mathbf{0}
\end{array}\right\rfloor,
$$

it can be shown that

$$
\mathbf{J N}=(\mathbf{J} \overline{\mathbf{N}})^{T} \text { or } \quad \mathbf{N}=\mathbf{J} \overline{\mathbf{N}}^{T} \mathbf{J},
$$

where the over bar denotes the complex conjugate that applies to the terms $i \beta$ and ih only. We then have

$$
\mathbf{N}-p \mathbf{I}=\mathbf{J}\left(\overline{\mathbf{N}}^{T}-p \mathbf{I}\right) \mathbf{J}
$$

so that the sextic equation for $p$ is

$$
|\mathbf{N}-p \mathbf{I}|=\left|\overline{\mathbf{N}}^{T}-p \mathbf{I}\right|=|\overline{\mathbf{N}}-p \mathbf{I}|=0 .
$$

It tells us that the sextic equation has no imaginary part. This completes the proof.

The $\xi$ in (2.21) is the right eigenvector. Let $\boldsymbol{\eta}$ be the left eigenvector of $\mathbf{N}$, i.e.

$$
\eta^{T} \mathbf{N}=p \eta^{T} \quad \text { or } \quad \mathbf{N}^{T} \eta=p \eta \text {. }
$$

Since the coefficients of the sextic equation in $p$ are real, if a complex $p$ is an eigenvalue so is its complex conjugate $\bar{p}$. Let

$$
\mathbf{N} \xi^{*}=\bar{p} \xi^{*}
$$

where $\xi^{*}$ is not necessarily identical to $\bar{\xi}$. Taking the complex conjugate of (3.6) and using (3.2) we have

$$
\mathbf{N}^{T}\left(\mathbf{J} \bar{\xi}^{*}\right)=p\left(\mathbf{J} \bar{\xi}^{*}\right)
$$

In view of $(3.5)_{2}$ we may take

$$
\eta=\mathbf{J} \bar{\xi}^{*}=\left\lfloor\begin{array}{l}
\overline{\mathbf{b}}^{*} \\
\overline{\mathbf{a}}^{*}
\end{array}\right\rfloor .
$$


Likewise, if $\eta^{*}$ is the left eigenvector associated with $\bar{p}$, we have

$$
\left(\eta^{*}\right)^{T} \mathbf{N}=\bar{p}\left(\eta^{*}\right)^{T} \text { or } \quad \mathbf{N}^{T} \eta^{*}=\bar{p} \eta^{*}
$$

Taking the complex conjugate of (2.21) and pre-multiplying the result by $\mathbf{J}$ we obtain

$$
\mathbf{J} \overline{\mathbf{N}} \bar{\xi}=\bar{p} \mathbf{J} \bar{\xi} .
$$

Again, use of (3.2) leads to

$$
\mathbf{N}^{T}(\mathbf{J} \bar{\xi})=\bar{p}(\mathbf{J} \bar{\xi})
$$

In view of $(3.9)_{2}$ we may take

$$
\eta^{*}=\mathbf{J} \bar{\xi}=\left\lfloor\begin{array}{l}
\overline{\mathbf{b}} \\
\overline{\mathbf{a}}
\end{array}\right\rfloor
$$

When $\beta=0$ and $h=0, \mathbf{a}^{*}, \mathbf{b}^{*}, \xi^{*}, \eta^{*}$ are $\overline{\mathbf{a}}, \overline{\mathbf{b}}, \bar{\xi}, \bar{\eta}$, respectively.

Assuming that all eigenvalues are complex, let $p_{n}(n=1,2,3)$ be the eigenvalues with a positive imaginary part. The associated right and left eigenvectors are $\xi_{n}$ and $\eta_{n}$ $(n=1,2,3)$. The remaining eigenvalues are $\bar{p}_{n}(n=1,2,3)$ and the associated right and left eigenvectors are $\xi_{n}^{*}$ and $\eta_{n}^{*}(n=1,2,3)$. The right and left eigenvectors associated with different eigenvalues are orthogonal to each other. We can normalize the eigenvectors such that

$$
\eta_{i}^{T} \xi_{j}=\delta_{i j}, \quad \eta_{i}^{T} \xi_{j}^{*}=0, \quad\left(\eta_{i}^{*}\right)^{T} \xi_{j}=0, \quad\left(\eta_{i}^{*}\right)^{T} \xi_{j}^{*}=\delta_{i j},
$$

where $\delta_{i j}$ is the Kronecker delta. Let

$$
\begin{aligned}
& \mathbf{A}=\left[\mathbf{a}_{1}, \mathbf{a}_{2}, \mathbf{a}_{3}\right], \quad \mathbf{B}=\left[\mathbf{b}_{1}, \mathbf{b}_{2}, \mathbf{b}_{3}\right], \\
& \mathbf{A}^{*}=\left[\begin{array}{lll}
\mathbf{a}_{1}^{*}, & \mathbf{a}_{2}^{*}, & \mathbf{a}_{3}^{*}
\end{array}\right], \quad \mathbf{B}^{*}=\left[\mathbf{b}_{1}^{*}, \mathbf{b}_{2}^{*}, \mathbf{b}_{3}^{*}\right]
\end{aligned}
$$

The orthogonality relations (3.13) can be written as, using (3.8) and (3.12),

$$
\left[\begin{array}{cc}
\left(\overline{\mathbf{B}}^{*}\right)^{T} & \left(\overline{\mathbf{A}}^{*}\right)^{T} \\
\overline{\mathbf{B}}^{T} & \overline{\mathbf{A}}^{T}
\end{array}\right]\left[\begin{array}{ll}
\mathbf{A} & \mathbf{A}^{*} \\
\mathbf{B} & \mathbf{B}^{*}
\end{array}\right]=\mathbf{I},
$$

or

$$
\begin{aligned}
& \left(\overline{\mathbf{B}}^{*}\right)^{T} \mathbf{A}+\left(\overline{\mathbf{A}}^{*}\right)^{T} \mathbf{B}=\mathbf{I}=\overline{\mathbf{B}}^{T} \mathbf{A}^{*}+\overline{\mathbf{A}}^{T} \mathbf{B}^{*}, \\
& \left(\overline{\mathbf{B}}^{*}\right)^{T} \mathbf{A}^{*}+\left(\overline{\mathbf{A}}^{*}\right)^{T} \mathbf{B}^{*}=\mathbf{0}=\overline{\mathbf{B}}^{T} \mathbf{A}+\overline{\mathbf{A}}^{T} \mathbf{B} .
\end{aligned}
$$


The two $6 \times 6$ matrices on the left of (3.15) are the inverse of each other. Their products commute so that

$$
\left\lfloor\begin{array}{ll}
\mathbf{A} & \mathbf{A}^{*} \\
\mathbf{B} & \mathbf{B}^{*}
\end{array}\right\rfloor\left[\begin{array}{cc}
\left(\overline{\mathbf{B}}^{*}\right)^{T} & \left(\overline{\mathbf{A}}^{*}\right)^{T} \\
\overline{\mathbf{B}}^{T} & \overline{\mathbf{A}}^{T}
\end{array}\right\rfloor=\mathbf{I},
$$

or

$$
\begin{aligned}
& \mathbf{A}\left(\overline{\mathbf{B}}^{*}\right)^{T}+\mathbf{A}^{*} \overline{\mathbf{B}}^{T}=\mathbf{I}=\mathbf{B}\left(\overline{\mathbf{A}}^{*}\right)^{T}+\mathbf{B}^{*} \overline{\mathbf{A}}^{T}, \\
& \mathbf{A}\left(\overline{\mathbf{A}}^{*}\right)^{T}+\mathbf{A}^{*} \overline{\mathbf{A}}^{T}=\mathbf{0}=\mathbf{B}\left(\overline{\mathbf{B}}^{*}\right)^{T}+\mathbf{B}^{*} \overline{\mathbf{B}}^{T} .
\end{aligned}
$$

These are the closure relations.

Extending the definition of Barnett-Lothe tensors [24, 25], let

$$
\begin{gathered}
\mathbf{S}=i\left[2 \mathbf{A}\left(\overline{\mathbf{B}}^{*}\right)^{T}-\mathbf{I}\right]=-i\left[2 \mathbf{A}^{*} \overline{\mathbf{B}}^{T}-\mathbf{I}\right], \\
\mathbf{H}=2 i \mathbf{A}\left(\overline{\mathbf{A}}^{*}\right)^{T}=-2 i \mathbf{A}^{*} \overline{\mathbf{A}}^{T}, \\
\mathbf{L}=-2 i \mathbf{B}\left(\overline{\mathbf{B}}^{*}\right)^{T}=2 i \mathbf{B}^{*} \overline{\mathbf{B}}^{T} .
\end{gathered}
$$

They are not necessarily real. It can be shown that

$$
\overline{\mathbf{H}}=\mathbf{H}^{T}, \quad \overline{\mathbf{L}}=\mathbf{L}^{T},
$$

so that $\mathbf{H}$ and $\mathbf{L}$ are hermitian. We can re-write (3.19) as

or

$$
\begin{aligned}
& \mathbf{S}=i\left[\mathbf{A}\left(\overline{\mathbf{B}}^{*}\right)^{T}-\mathbf{A}^{*} \overline{\mathbf{B}}^{T}\right], \\
& \mathbf{H}=i\left[\mathbf{A}\left(\overline{\mathbf{A}}^{*}\right)^{T}-\mathbf{A}^{*} \overline{\mathbf{A}}^{T}\right], \\
& \mathbf{L}=-i\left[\mathbf{B}\left(\overline{\mathbf{B}}^{*}\right)^{T}-\mathbf{B}^{*} \overline{\mathbf{B}}^{T}\right],
\end{aligned}
$$

$$
\left[\begin{array}{cc}
\mathbf{S} & \mathbf{H} \\
-\mathbf{L} & \overline{\mathbf{S}}^{T}
\end{array}\right]=\left[\begin{array}{cc}
\mathbf{A} & \mathbf{A}^{*} \\
\mathbf{B} & \mathbf{B}^{*}
\end{array}\right]\left[\begin{array}{cc}
i \mathbf{I} & \mathbf{0} \\
\mathbf{0} & -i \mathbf{I}
\end{array}\right]\left[\begin{array}{cc}
\left(\overline{\mathbf{B}}^{*}\right)^{T} & \left(\overline{\mathbf{A}}^{*}\right)^{T} \\
\overline{\mathbf{B}}^{T} & \overline{\mathbf{A}}^{T}
\end{array}\right] .
$$

It is readily shown that, using (3.15) and (3.17),

$$
\left\lfloor\begin{array}{cc}
\mathbf{S} & \mathbf{H} \\
-\mathbf{L} & \overline{\mathbf{S}}^{T}
\end{array}\right\rfloor\left[\begin{array}{cc}
\mathbf{S} & \mathbf{H} \\
-\mathbf{L} & \overline{\mathbf{S}}^{T}
\end{array}\right\rfloor=-\left\lfloor\begin{array}{cc}
\mathbf{I} & \mathbf{0} \\
\mathbf{0} & \mathbf{I}
\end{array}\right\rfloor,
$$

or

$$
\begin{aligned}
& \mathbf{H L}-\mathbf{S S}=\mathbf{I}=\mathbf{L H}-\overline{\mathbf{S}}^{T} \overline{\mathbf{S}}^{T}, \\
& \mathbf{S H}+\mathbf{H} \overline{\mathbf{S}}^{T}=\mathbf{0}=\mathbf{L S}+\overline{\mathbf{S}}^{T} \mathbf{L} .
\end{aligned}
$$


If $\mathbf{H}$ and $\mathbf{L}$ are non-singular, the last two equations in (3.24) can be written as

$$
\mathbf{H}^{-1} \mathbf{S}+\overline{\mathbf{S}}^{T} \mathbf{H}^{-1}=\mathbf{0}=\mathbf{S L}^{-1}+\mathbf{L}^{-1} \overline{\mathbf{S}}^{T} .
$$

It can be shown that $\mathbf{S H}, \mathbf{L S}, \mathbf{H}^{-1} \mathbf{S}$ and $\mathbf{S L}^{-1}$ are skew-hermitian. If they are real, they are skew-symmetric.

When $\mathbf{A}$ is non-singular, we write the last equation in (3.16) as

$$
\left(\overline{\mathbf{B}} \overline{\mathbf{A}}^{-1}\right)^{T}+\mathbf{B} \mathbf{A}^{-1}=\mathbf{0} .
$$

Hence the impedance tensor

$$
\mathbf{M}=-i \mathbf{B} \mathbf{A}^{-1}
$$

is hermitian. Writing (3.27) as

$$
\mathbf{M}=-i\left(\overline{\mathbf{A}}^{*} \mathbf{B}^{T}\right)^{T}\left[\mathbf{A}\left(\overline{\mathbf{A}}^{*}\right)^{T}\right]^{-1}
$$

and using (3.19) we obtain

$$
\mathbf{M}=\mathbf{H}^{-1}(\mathbf{I}+i \mathbf{S}),
$$

where use has been made of $(3.25)_{1}$.

\section{Rayleigh surface waves}

\subsection{General anisotropic materials.}

The stresses computed from (2.7) using (2.15), (2.19) 1 and (2.24) are

$$
\begin{gathered}
\sigma_{i 1}=i k\left[-(p+i \beta) b_{i}+X a_{i}+i Y_{i k} a_{k}\right] e^{i k\left[x_{1}-v t+(p+i \beta) x_{2}\right]} \\
\sigma_{i 2}=i k b_{i} e^{i k\left[x_{1}-v t+(p+i \beta) x_{2}\right]}
\end{gathered}
$$

On the other hand, the stresses obtained from (2.10) using (2.15) and (2.19) 1 are

$$
\begin{aligned}
& \sigma_{i 1}=i k\left[Q_{i k}+(p-i \beta) R_{i k}\right] a_{k} e^{i k\left[x_{1}-v t+(p+i \beta) x_{2}\right]}, \\
& \sigma_{i 2}=i k\left[R_{k i}+(p-i \beta) T_{i k}\right] a_{k} e^{i k\left[x_{1}-v t+(p+i \beta) x_{2}\right]} .
\end{aligned}
$$

Equations (4.1) and (4.2) are consistent if

$$
\mathbf{b}=\left[\mathbf{R}^{T}+(p-i \beta) \mathbf{T}\right] \mathbf{a},
$$

and

$$
\begin{gathered}
(\mathbf{D}-i \mathbf{W}) \mathbf{a}=\mathbf{0} \\
\mathbf{D}=\mathbf{Q}-X \mathbf{I}+\beta^{2} \mathbf{T}+p\left(\mathbf{R}+\mathbf{R}^{T}\right)+p^{2} \mathbf{T} .
\end{gathered}
$$


In (4.4), $\mathbf{W}$ is an anti-symmetric matrix

$$
\mathbf{W}=\beta\left(\mathbf{R}-\mathbf{R}^{T}\right)+h \mathbf{Y}=\left[\begin{array}{ccc}
0 & w_{3} & -w_{2} \\
-w_{3} & 0 & w_{1} \\
w_{2} & -w_{1} & 0
\end{array}\right]
$$

For a non-trivial solution of a from (4.4) the determinant of (D-iW) must vanish. It can be shown ([21], p.23) that

$$
|\mathbf{D}-i \mathbf{W}|=|\mathbf{D}|-\mathbf{w}^{T} \mathbf{D} \mathbf{w}=0,
$$

where, from (4.6),

$$
\mathbf{w}=\left\lfloor\begin{array}{c}
w_{1} \\
w_{2} \\
w_{3}
\end{array}\right\rfloor=\left\lfloor\begin{array}{c}
\beta\left(C_{46}-C_{25}\right) \\
\beta\left(C_{56}-C_{24}\right) \\
\beta\left(C_{12}-C_{66}\right)+h
\end{array}\right\rfloor,
$$

and $C_{\alpha \beta}$ is the contracted notation of $C_{i j k s}$. Equation (4.7) provides an alternate proof that the coefficients of the sextic equation in $p$ are real [18].

For a surface wave propagating in the direction of the $x_{1}$-axis in the half space $x_{2} \geq 0$, the surface traction at $x_{2}=0$ must vanish. The surface traction $\sigma_{i 2}$ at $x_{2}=0$ can be obtained by superimposing three solutions of $(4.1)_{2}$ associated with three $p_{n}(n=1,2,3)$. We have

$$
\left(\sigma_{i 2}\right)_{x_{2}=0}=i k(\mathbf{B q})_{i} e^{i k\left(x_{1}-v t\right)}
$$

where $\mathbf{q}$ is a constant to be determined. Likewise, the displacement at $x_{2}=0$ can be obtained by superimposing three solutions of (2.19) $)_{1}$ associated with three $p_{n}(n=1,2,3)$. The result is

$$
(\mathbf{u})_{x_{2}=0}=\mathbf{A q} e^{i k\left(x_{1}-v t\right)} .
$$

The surface traction vanishes if

$$
\mathbf{B q}=\mathbf{0} \text {. }
$$

Equation (4.10) has a non-trivial solution for $\mathbf{q}$ if

$$
|\mathbf{B}|=0 \text {. }
$$

This is the secular equation for the wave speed $v$. 
As in the case of homogeneous materials without the influence of gravity, (4.11) is not the only secular equation available [20, 21]. From (3.19), (3.27) and (3.29) alternate secular equations are

$$
|\mathbf{L}|=0, \quad|\mathbf{M}|=0, \quad|\mathbf{I}+i \mathbf{S}|=0 .
$$

The secular equations are the vanishing of a $3 \times 3$ determinant for general anisotropic materials and $2 \times 2$ determinant for orthotropic materials.

Equation (4.9) shows that there are three components for the surface wave solution associated with $q_{1}, q_{2}, q_{3}$. If the surface traction can vanish by superimposing two solutions, we have a two-component surface wave. Surface waves in an orthotropic elastic material are two-component surface waves. There are special anisotropic elastic materials for which a surface wave can propagate with only one component in a homogenous material without the influence of gravity [26, 27]. It is shown in [18] that one-component surface waves can propagate in an exponentially graded anisotropic material under the influence of gravity.

It should be pointed out that the surface wave solutions for anisotropic elastic halfspace with exponentially graded materials under the influence of gravity involve two parameters $\beta=\alpha / k$ and $h=\rho g / k . \quad \beta=0$ when $\alpha=0$, which means that the material is homogeneous. However, $\beta=0$ also when $k=\infty$. Likewise, $h=0$ when there is no gravitation, but $h=0$ also when $k=\infty$. Thus the solution recovers the solution for a homogeneous material without the influence of gravity when the wave number $k$ is infinity. It tells us that, if a surface wave exists for a homogeneous material without the influence of gravity, it exists also for the exponentially graded elastic material of general anisotropy under the influence of gravity when the wave number $k$ is very large. It also tells us that, as $k \rightarrow \infty$, the surface wave speed approaches the surface wave speed for the homogeneous material without the influence of gravity.

We next apply the above results to orthotropic materials.

\subsection{Orthotropic materials.}

In the special case of orthotropic materials we can ignore the anti-plane deformation. For the in-plane deformation (4.4) has a non-trivial solution for $\mathbf{a}$ if 


$$
|\mathbf{D}-i \mathbf{W}|=\left|\begin{array}{cc}
C_{11}-X+C_{66} \beta^{2}+C_{66} p^{2} & \left(C_{12}+C_{66}\right) p-i\left[\left(C_{12}-C_{66}\right) \beta+h\right] \\
\left(C_{12}+C_{66}\right) p+i\left[\left(C_{12}-C_{66}\right) \beta+h\right] & C_{66}-X+C_{22} \beta^{2}+C_{22} p^{2}
\end{array}\right|=0 .
$$

By inspection, it is obvious that (4.13) is a quadratic equation in $p^{2}$ with real coefficients. We have

$$
\begin{gathered}
p^{4}-E p^{2}+P=0, \\
P=\left\{\left(C_{11}-X+C_{66} \beta^{2}\right)\left(C_{66}-X+C_{22} \beta^{2}\right)-\left[\left(C_{12}-C_{66}\right) \beta+h\right]^{2}\right\} /\left(C_{22} C_{66}\right) . \\
E=-\left[C_{22}\left(G_{0}-X\right)-C_{66}\left(2 C_{12}+X\right)\right] /\left(C_{22} C_{66}\right)-2 \beta^{2},
\end{gathered}
$$

where

$$
G_{0}=\left(C_{11} C_{22}-C_{12}^{2}\right) / C_{22} .
$$

When $h=0$, this recovers the eigenrelation given in [3]. If $p_{1}$ and $p_{2}$ are the eigenvalues with a positive imaginary part,

$$
p_{1}^{2}+p_{2}^{2}=E, \quad p_{1} p_{2}=-\sqrt{P} .
$$

Hence

$$
p_{1}+p_{2}=i \sqrt{2 \sqrt{P}-E} .
$$

$P>0$ when $p_{1}$ and $p_{2}$ are complex. If one of $p_{1}, p_{2}$ is purely imaginary while the other one is real, $P<0$. This can happen to one-component waves and supersonic waves. We do not consider $P<0$ here.

To derive the secular equation using (4.11) we need to find the eigenvectors $\mathbf{b}_{1}$ and $\mathbf{b}_{2}$ associated with $p_{1}$ and $p_{2}$. The matrix $\mathbf{B}=\left[\mathbf{b}_{1}, \mathbf{b}_{2}\right]$ is a $2 \times 2$ matrix. One way is to compute b from (4.3) after finding a from (4.4). This approach would lead to a polynomial in $p$ of degree three for $\mathbf{b}$. Another way is to find the eigenvector $\xi$ from (2.21). For orthotropic materials, (2.21) has the expression

$$
(\mathbf{N}-p \mathbf{I}) \xi=\left[\begin{array}{cccc}
-(p-i \beta) & -1 & 1 / C_{66} & 0 \\
-C_{12} / C_{22} & -(p-i \beta) & 0 & 1 / C_{22} \\
X-G_{0} & i h & -(p+i \beta) & -C_{12} / C_{22} \\
-i h & X & -1 & -(p+i \beta)
\end{array}\right]\left[\begin{array}{l}
a_{1} \\
a_{2} \\
b_{1} \\
b_{2}
\end{array}\right]=\mathbf{0} .
$$


Let $\mathbf{K}^{\boldsymbol{T}}$ be the adjoint matrix of $(\mathbf{N}-p \mathbf{I})$. This means that $K_{i j}$ is the cofactor of $(\mathbf{N}-p \mathbf{I})_{i j}$. We can choose

$$
\mathbf{a}=\vartheta\left[\begin{array}{l}
K_{i 1} \\
K_{i 2}
\end{array}\right], \quad \mathbf{b}=\vartheta\left[\begin{array}{l}
K_{i 3} \\
K_{i 4}
\end{array}\right],
$$

in which $\gamma$ is an arbitrary factor and $i=1,2,3$ or 4 . If we choose $\gamma=-1$ and $i=2$ we have

$$
\mathbf{b}=\left\lfloor\begin{array}{c}
i h p^{2}+G_{1} p+i G_{2} \\
X p^{2}-G_{3}
\end{array}\right\rfloor,
$$

where

$$
\begin{gathered}
G_{1}=G_{0}-\left(1+C_{12} / C_{22}\right) X, \\
G_{2}=\beta\left[G_{0}-\left(1-C_{12} / C_{22}\right) X\right]+h\left(\beta^{2}-C_{12} / C_{22}\right), \\
G_{3}=\left(G_{0}-X\right)\left(1-X / C_{66}\right)-\beta^{2} X+h\left(2 \beta-h / C_{66}\right) .
\end{gathered}
$$

There is no need to normalize the eigenvector in computing the secular equation, which is

$$
|\mathbf{B}|=\left|\begin{array}{cc}
i h p_{1}^{2}+G_{1} p_{1}+i G_{2} & i h p_{2}^{2}+G_{1} p_{2}+i G_{2} \\
X p_{1}^{2}-G_{3} & X p_{2}^{2}-G_{3}
\end{array}\right|=0,
$$

or, after using (4.17) and deleting the common factor $\left(p_{1}-p_{2}\right)$,

$$
G_{1}\left(X \sqrt{P}-G_{3}\right)+\left(X G_{2}+h G_{3}\right) \sqrt{2 \sqrt{P}-E}=0 .
$$

We will not consider the degenerate case $p_{1}=p_{2}$ here. The degenerate case can be studied separately [28, 29]. It should be noted that (4.23) is real even though the eigenvectors $\mathbf{b}_{1}$ and $\mathbf{b}_{2}$ employed in computing (4.23) are complex. It should also be noted that the secular equation (4.23) is fully explicit. The $X$, which is $\rho v^{2}$, is the only unknown. The wave is dispersive because it depends on the wave number $k$. In the special case $h=0,(4.23)$ reduces to (2.15) in [3].

When the material is homogeneous without the influence of gravity, $\beta=h=G_{2}=0$ so that (4.23) simplifies to

$$
X \sqrt{P}-G_{3}=0
$$

or

$$
C_{22} X \sqrt{C_{66}\left(C_{11}-X\right)}=\left(C_{11} C_{22}-C_{12}^{2}-C_{22} X\right) \sqrt{C_{22}\left(C_{66}-X\right)} .
$$


This recovers the secular equation $(12.10-17)$ in ([21], p. 481). It is a cubic equation in $X$ when both sides of (4.25) are squared.

\section{Stoneley waves}

\subsection{General anisotropic materials.}

Interfacial waves in two joined elastic half-spaces are known as Stoneley waves [30]. The analyses presented so far apply to the half-space $x_{2} \geq 0$. The same analyses apply to the half-space $x_{2} \leq 0$ if we replace $p$ by its complex conjugate $\bar{p}$ because the displacement and the stress must vanish at $x_{2}=-\infty$. The eigenvectors associated with $\bar{p}$ are, according to (3.6), $\xi^{*}=\left(\mathbf{a}^{*}, \mathbf{b}^{*}\right)$. For the Stoneley waves, the displacement and the surface traction at $x_{2}=0$ must be continuous.

The surface traction and displacement at $x_{2}=0$ for the half-space $x_{2} \geq 0$ are given in (4.9a,b). We write $(4.9 \mathrm{a}, \mathrm{b})$ are

$$
\begin{gathered}
\left(\sigma_{i 2}\right)_{x_{2}=0}=i k\left(\mathbf{B}_{(1)} \mathbf{q}_{(1)}\right)_{i} e^{i k\left(x_{1}-v t\right)}, \\
(\mathbf{u})_{x_{2}=0}=\mathbf{A}_{(1)} \mathbf{q}_{(1)} e^{i k\left(x_{1}-v t\right)},
\end{gathered}
$$

where the subscript (1) denotes the material in the half-space $x_{2} \geq 0$. The surface traction and displacement at $x_{2}=0$ for the half-space $x_{2} \leq 0$ are

$$
\begin{gathered}
\left(\sigma_{i 2}\right)_{x_{2}=0}=i k\left(\mathbf{B}_{(2)}^{*} \mathbf{q}_{(2)}\right)_{i} e^{i k\left(x_{1}-v t\right)}, \\
(\mathbf{u})_{x_{2}=0}=\mathbf{A}_{(2)}^{*} \mathbf{q}_{(2)} e^{i k\left(x_{1}-v t\right)},
\end{gathered}
$$

where the subscript (2) denotes the material in the half-space $x_{2} \leq 0$. The continuity of surface traction and displacement at $x_{2}=0$ demands that

$$
\mathbf{B}_{(1)} \mathbf{q}_{(1)}=\mathbf{B}_{(2)}^{*} \mathbf{q}_{(2)}, \quad \mathbf{A}_{(1)} \mathbf{q}_{(1)}=\mathbf{A}_{(2)}^{*} \mathbf{q}_{(2)} .
$$

Equation (3.27) for the material (1) can be written as

$$
\mathbf{B}_{(1)} \mathbf{q}_{(1)}=i \mathbf{M}_{(1)} \mathbf{A}_{(1)} \mathbf{q}_{(1)} \text {. }
$$

The corresponding equation for the material (2) is

$$
\mathbf{B}_{(2)}^{*} \mathbf{q}_{(2)}=-i \mathbf{M}_{(2)}^{*} \mathbf{A}_{(2)}^{*} \mathbf{q}_{(2)},
$$

where 


$$
\mathbf{M}_{(2)}^{*}=i \mathbf{B}_{(2)}^{*}\left(\mathbf{A}_{(2)}^{*}\right)^{-1}
$$

Imposition of the continuity condition (5.3) leads to

$$
\left[\mathbf{M}_{(1)}+\mathbf{M}_{(2)}^{*}\right] \mathbf{A}_{(1)} \mathbf{q}_{(1)}=\mathbf{0}
$$

A non-trivial solution for $\mathbf{A}_{(1)} \mathbf{q}_{(1)}$ exists if

$$
\left|\mathbf{M}_{(1)}+\mathbf{M}_{(2)}^{*}\right|=0 \text {. }
$$

This is the secular equation for a Stoneley wave to propagate. When $\beta=0$ and $h=0$, $\mathbf{M}_{(2)}^{*}=\overline{\mathbf{M}}_{(2)}$ and (5.7) recovers the secular equation for Stoneley waves in homogeneous materials without the influence of gravity [31]. Since the matrix $\mathbf{M}_{(1)}+\mathbf{M}_{(2)}^{*}$ is hermitian, its determinant is real. Thus the secular equation (5.7) has no imaginary part. Again, (5.7) for Stoneley waves is the vanishing of a $3 \times 3$ determinant for general anisotropic materials and $2 \times 2$ determinant for orthotropic materials. We discuss the latter below.

\subsection{Orthotropic materials.}

In the special case of orthotropic materials we will show below that the secular equation (5.7) is explicit in which the wave speed $v$ is the only unknown.

For the material (1) we have to compute $\mathbf{M}=-i \mathbf{B} \mathbf{A}^{-1}$ from (3.27). This means that we have to find the matrices $\mathbf{A}=\left[\mathbf{a}_{1}, \mathbf{a}_{2}\right]$ and $\mathbf{B}=\left[\mathbf{b}_{1}, \mathbf{b}_{2}\right]$ and the inverse of $\mathbf{A}$. They are all $2 \times 2$ matrices. Since the inverse of the matrix $\mathbf{A}$ is required, it is best if the elements of A have simple expressions. Again, there is no need to normalize the eigenvectors in computing $\mathbf{B} \mathbf{A}^{-1}$. Choosing $i=3$ and $\gamma=-C_{22} C_{66}$ in (4.19) we have,

$$
\mathbf{a}=\left[\begin{array}{c}
C_{22} p^{2}+f \\
-(n p+i m)
\end{array}\right], \quad \mathbf{b}=\left[\begin{array}{c}
e_{2} p^{3}-i e_{2} \beta p^{2}-d_{1} p-i d_{0} \\
-e_{2} p^{2}+i e_{1} p+e_{0}
\end{array}\right],
$$

where

$$
\begin{gathered}
f=C_{66}-X+C_{22} \beta^{2}, \quad n=C_{12}+C_{66}, \quad m=\left(C_{12}-C_{66}\right) \beta+h, \\
d_{0}=C_{66}(\beta f+m), \quad d_{1}=C_{66}(n-f), \\
e_{0}=C_{12} f-C_{22} \beta m, \quad e_{1}=C_{22}(\beta n-m), \quad e_{2}=C_{22} C_{66} .
\end{gathered}
$$

Hence 


$$
\begin{gathered}
\mathbf{A}=\left\lfloor\begin{array}{cc}
C_{22} p_{1}^{2}+f & C_{22} p_{2}^{2}+f \\
-\left(n p_{1}+i m\right) & -\left(n p_{2}+i m\right)
\end{array}\right\rfloor, \\
\mathbf{B}=\left\lfloor\begin{array}{cc}
e_{2} p_{1}^{3}-i e_{2} \beta p_{1}^{2}-d_{1} p_{1}-i d_{0} & e_{2} p_{2}^{3}-i e_{2} \beta p_{2}^{2}-d_{1} p_{2}-i d_{0} \\
-e_{2} p_{1}^{2}+i e_{1} p_{1}+e_{0} & -e_{2} p_{2}^{2}+i e_{1} p_{2}+e_{0}
\end{array}\right\rfloor .
\end{gathered}
$$

It can be shown that

$$
\begin{gathered}
|\mathbf{A}|=\left(p_{1}-p_{2}\right)\left(F+F_{0}\right), \\
F=n\left(C_{22} \sqrt{P}+f\right), \quad F_{0}=m C_{22} \sqrt{K}, \\
K=2 \sqrt{P}-E .
\end{gathered}
$$

in which use have been made of (4.17). Therefore

$$
\mathbf{A}^{-1}=\frac{1}{|\mathbf{A}|}\left\lfloor\begin{array}{cc}
-\left(n p_{2}+i m\right) & -\left(C_{22} p_{2}^{2}+f\right) \\
n p_{1}+i m & C_{22} p_{1}^{2}+f
\end{array}\right\rfloor .
$$

The impedance tensor for $\mathbf{M}$ given in (3.27) can now be computed. The result is

where

$$
M_{11}=\frac{U+U_{0}}{F+F_{0}}, \quad M_{22}=\frac{V+V_{0}}{F+F_{0}} \quad M_{21}=-M_{12}=i \frac{Z+Z_{0}}{F+F_{0}},
$$

$$
\begin{gathered}
U=e_{2}(n \sqrt{P}-\beta m) \sqrt{K}, \quad U_{0}=e_{2}(m-\beta n) \sqrt{P}+m\left(d_{1}-e_{2} E\right)-d_{0} n, \\
V=\left(e_{2} f+e_{0} C_{22}\right) \sqrt{K}, \quad V_{0}=-e_{1}\left(f+C_{22} \sqrt{P}\right), \\
Z=e_{2} n \sqrt{P}-\left(e_{0} n+e_{1} m\right), \quad Z_{0}=e_{2} m \sqrt{K} .
\end{gathered}
$$

$M_{11}$ and $M_{22}$ are real while $M_{21}=-M_{12}$ are purely imaginary, proving that $\mathbf{M}$ is indeed hermitian.

For material (2), we have to compute $\mathbf{M}^{*}$ from (5.5). The derivations from (5.8) through (5.14) for material (1) hold for material (2) if we replace $p_{1}, p_{2}$ by the complex conjugate $\bar{p}_{1}, \bar{p}_{2}$. The result is

$$
M_{11}^{*}=\frac{U-U_{0}}{F-F_{0}}, \quad M_{22}^{*}=\frac{V-V_{0}}{F-F_{0}} \quad M_{21}^{*}=-M_{12}^{*}=-i \frac{Z-Z_{0}}{F-F_{0}} .
$$

Again, $M_{11}^{*}$ and $M_{22}^{*}$ are real while $M_{21}^{*}=-M_{12}^{*}$ are purely imaginary, proving that $\mathbf{M}^{*}$ is hermitian. 
With (5.13) and (5.15), the secular equation (5.7) is

$$
\begin{aligned}
\left\lfloor\left(\frac{U+U_{0}}{F+F_{0}}\right)_{(1)}+\left(\frac{U-U_{0}}{F-F_{0}}\right)_{(2)}\right\rfloor & \left.\mid\left(\frac{V+V_{0}}{F+F_{0}}\right)_{(1)}+\left(\frac{V-V_{0}}{F-F_{0}}\right)_{(2)}\right\rfloor \\
- & {\left[\left(\frac{Z+Z_{0}}{F+F_{0}}\right)_{(1)}-\left(\frac{Z-Z_{0}}{F-F_{0}}\right)_{(2)}\right]^{2}=0 . }
\end{aligned}
$$

This is an explicit secular equation. It has no imaginary parts. The only unknown in the secular equation is the wave speed $v$, which is implicit in $X_{(1)}$ and $X_{(2)}$ where $X_{(1)}=\rho_{(1)} v^{2}$ and $X_{(2)}=\rho_{(2)} v^{2}$. Different secular equations have been presented in [16, 17] where the secular equations are the vanishing of a $4 \times 4$ determinant. They are not as explicit as (5.16). Moreover, as we will show below, (5.16) recovers easily the secular equation for the special case of homogeneous isotropic materials without gravity.

When the material is homogeneous without the influence of gravity, $\beta=h=0$ so that $m=d_{0}=e_{1}=0 . F_{0}, U_{0}, V_{0}$ and $Z_{0}$ all vanish. The secular equation (5.16) simplifies to

$$
\left[\left(\frac{U}{F}\right)_{(1)}+\left(\frac{U}{F}\right)_{(2)} \rrbracket\left(\frac{V}{F}\right)_{(1)}+\left(\frac{V}{F}\right)_{(2)}\right]-\left[\left(\frac{Z}{F}\right)_{(1)}-\left(\frac{Z}{F}\right)_{(2)}\right]^{2}=0,
$$

where

$$
\begin{gathered}
\frac{U}{F}=\frac{C_{66} \sqrt{C_{22}\left(C_{11}-X\right)} \sqrt{K}}{\sqrt{C_{22}\left(C_{11}-X\right)}+\sqrt{C_{66}\left(C_{66}-X\right)}}, \\
\frac{V}{F}=\frac{C_{22} \sqrt{C_{66}\left(C_{66}-X\right)} \sqrt{K}}{\sqrt{C_{22}\left(C_{11}-X\right)}+\sqrt{C_{66}\left(C_{66}-X\right)}}, \\
\frac{Z}{F}=\frac{C_{66} \sqrt{C_{22}\left(C_{11}-X\right)}-C_{12} \sqrt{C_{66}\left(C_{66}-X\right)}}{\sqrt{C_{22}\left(C_{11}-X\right)}+\sqrt{C_{66}\left(C_{66}-X\right)}}, \\
K=\frac{1}{C_{22} C_{66}}\left[\left(\sqrt{C_{22}\left(C_{11}-X\right)}+\sqrt{C_{66}\left(C_{66}-X\right)}\right)^{2}-\left(C_{12}+C_{66}\right)^{2}\right] .
\end{gathered}
$$

With (5.18), (5.17) provides an explicit secular equation for Stoneley waves in two homogeneous orthotropic half-spaces without the influence of gravity.

It should be noted that, for orthotropic materials, only the elastic stiffness $C_{11}, C_{12}$, $C_{22}$ and $C_{66}$ are needed in the secular equations. No simplification is gained for hexagonal materials if $x_{1}$ or $x_{2}$ is the symmetry axis. 
For cubic materials we set $C_{11}=C_{22}$ in the secular equations.

For isotropic materials (also for hexagonal materials with $x_{3}$ being the symmetry axis) we impose the conditions $C_{11}=C_{22}$ and $2 C_{66}=C_{11}-C_{12}$. Equation (5.18) simplifies to

$$
\begin{gathered}
\frac{U}{F}=\frac{C_{11} C_{66} \Delta(\Delta+\delta)}{C_{11} \Delta+C_{66} \delta}=\frac{X \Delta}{1-\Delta \delta}, \\
\frac{V}{F}=\frac{C_{11} C_{66} \delta(\Delta+\delta)}{C_{11} \Delta+C_{66} \delta}=\frac{X \delta}{1-\Delta \delta}, \\
\frac{Z}{F}=\frac{C_{66}\left[C_{11} \Delta-\left(C_{11}-2 C_{66}\right) \delta\right]}{C_{11} \Delta+C_{66} \delta}=2 C_{66}-\frac{X}{1-\Delta \delta},
\end{gathered}
$$

where

$$
\Delta=\sqrt{1-\left(X / C_{11}\right)}, \quad \delta=\sqrt{1-\left(X / C_{66}\right)} .
$$

The $(U / F),(V / F),(Z / F)$ in (5.19) are the elements of the $2 \times 2$ hermitian matrix M. The first equalities in (5.19) are deduced from (5.18) that were computed from the $\mathbf{M}$ shown in (3.27). The second equalities in (5.19) are obtained from the first equalities by multiplying the denominator and the numerator by $(\Delta-\delta)$. They recover the expressions of $\mathbf{M}$ given in [31] who employed the $\mathbf{M}$ shown in (3.29) in which the Barnett-Lothe tensors $\mathbf{H}$ and $\mathbf{S}$ are computed from the integral formalism [24]. Let

$$
\kappa=1-\Delta \delta, \quad R=[2-(X / \mu)]^{2}-4 \Delta \delta, \quad \mu=C_{66} .
$$

Substituting the second expressions of $(U / F),(V / F),(Z / F)$ in (5.19) into (5.17) and deleting the non-zero common factor $-\left(\kappa_{1} \kappa_{2}\right)^{-1}$ we obtain

$$
\begin{aligned}
& \mu_{1}^{2} R_{1} \kappa_{2}+\mu_{2}^{2} R_{2} \kappa_{1}-X_{1} X_{2}\left(\Delta_{1} \delta_{2}+\Delta_{2} \delta_{1}\right) \\
& \quad-2 \mu_{1} \mu_{2}[2 \kappa-(X / \mu)]_{1}[2 \kappa-(X / \mu)]_{2}=0 .
\end{aligned}
$$

In the above, the subscripts 1 and 2 refer to materials (1) and (2), respectively. This recovers the explicit secular equation for Stoneley waves in two homogeneous isotropic elastic half-spaces presented in [31 - 33].

We introduced $R$ in the secular equation (5.22) for Stoneley waves to call readers' attention that $R=0$ is a secular equation for Rayleigh waves [33]. A Stoneley wave becomes a Rayleigh wave when material (2) in the half-space $x_{2} \leq 0$ is a void. In this case $\mathbf{M}_{(2)}^{*}=\mathbf{0}$, and the secular equation (5.7) for Stoneley waves reduces to the secular 
equation $(4.12)_{2}$ for Rayleigh waves. In the special case of homogeneous isotropic materials without the gravity, $\mathbf{M}_{(2)}^{*}=\mathbf{0}$ means that all terms in (5.22) vanish except the first term. The vanishing of the first term demands that $R_{1}=0$ or, from $(5.21)_{2}$,

$$
\left[2-\left(X / C_{66}\right)\right]^{2}-4 \sqrt{1-\left(X / C_{11}\right)} \sqrt{1-\left(X / C_{66}\right)}=0,
$$

for material (1) in the half-space $x_{2} \geq 0$. This is one of the expressions for the secular equation for Rayleigh waves ([21], p.482). If we multiply (5.23a) by $(\Delta+\delta)$ we obtain

$$
\left(X / C_{66}\right) \sqrt{1-\left(X / C_{11}\right)}-\left[4\left(1-C_{66} / C_{11}\right)-X\right] \sqrt{1-\left(X / C_{66}\right)}=0 .
$$

This is another expression for the secular equation for Rayleigh waves in homogeneous isotropic elastic half-space ([21], p.482). Equation (5.23b) can be deduced from the secular equation (4.25) for orthotropic materials by specializing it to isotropic materials.

\section{Concluding remarks}

We have extended the analysis presented in the literature for Rayleigh waves and Stoneley waves in exponentially graded orthotropic elastic materials with the influence of gravity to general anisotropic elastic materials. The analysis involve two parameters $\beta=\alpha / k$ and $h=\rho g / k$ that are related to the inhomogeneity of the materials and the influence of gravity, respectively. When $\beta=0$ and $h=0$, all equations recover the known equations in the literature for homogeneous anisotropic elastic materials without the influence of gravity. Since $\beta=0$ and $h=0$ when the wave number $k \rightarrow \infty$, if a Rayleigh wave exists for homogeneous anisotropic elastic materials without the influence of gravity, a Rayleigh wave exists for the exponentially graded elastic materials of general anisotropy with the influence of gravity at least for a very large $k$. The same can be said of the Stoneley wave.

We presented secular equations for Rayleigh wave speed and Stoneley wave speed for exponentially graded elastic materials of general anisotropy with the influence of gravity. For the special case of orthotropic materials, explicit secular equations are obtained in (4.23) for Rayleigh waves and in (5.16) for Stoneley waves. The corresponding secular equations when the materials are homogeneous without the influence of gravity are deduced in (4.24) and (5.17), respectively. 
It is known that one-component surface wave can propagate in a special homogenous anisotropic elastic material without the influence of gravity [26, 27]. It is also known that one-components Stoneley wave can propagate in an anisotropic elastic bimaterial [34]. The problem of one-component surface waves in an exponentially graded anisotropic material with the influence of gravity has been addressed in [18]. The question is open if one-component Stoneley waves can propagate in an exponentially graded anisotropic bimaterial under the influence of gravity.

\section{References}

1. Pal, P.K., Acharya, D.: Effects of inhomogeneity on surface waves in anisotropic media. Sadhana 23, 247-258 (1998).

2. Collet, Bernard, Destrade, Michael, Maugin, Gerard: Bleustein-Gulyaev waves in some functionally graded materials. European Journal of Mechanics, 25, 695-706 (2006).

3. Destrade, M.: Seismic Rayleigh waves on an exponentially graded, orthotropic elastic half-space. Proc. Roy. Soc. London A463, 495-502 (2007).

4. Kulkarni, S.S., Achenbach, J.D.: Application of reciprocity theorem to determine line-loaded generated surface waves on an inhomogeneous transversely isotropic half-space. Wave Motion 45, 350-360 (2008).

5. Qian, Z. H., Jin, F., Lu, T. J., Kishimoto, K.: Transverse surface waves in functionally graded piezoelectric materials with exponential variation. Smart Materials \& Structures 17(6), Article Number 065005 (2008).

6. Eskandari, M., Shodja, H. M.: Love waves propagation in functionally graded piezoelectric materials with quadratic variation. J. Sound and Vibration, 313(1-2), 195-204 (2008).

7. Vinh, Pham Chi, Seriani, Geza: Explicit secular equations of Rayleigh waves in a non-homogeneous orthotropic elastic medium under the influence of gravity. Wave Motion 46, 427-434 (2009).

8. Cao, X. S., Jin, F., Wang, Z. K., Lu, T. J.: Bleustein-Gulyaev waves in a functionally graded piezoelectric material layered structure. Science in China, Series G-Physics Mechanics \& Astronomy 52(4), 613-625 (2009). 
9. Qian, Z. H., Jin, F., Lu, T. J., Kishimoto, K.: Transverse surface waves in a functionally graded piezoelectric substrate coated with a finite-thickness metal waveguide layer. Appl. Physics Letter 94(2), Article Number 023501 (2009).

10. Shuvalov, A. L., Poncelet, O., Golkin, S. V.: Existence and spectral properties of shear horizontal surface acoustic waves in vertically periodic half-spaces. Proc. R. Soc. London A465, 1489-1511 (2009).

11. Achenbach, Jan D., Balogun, Oluwaseyi: Anti-plane surface waves on a half-space with depth-dependent properties. Wave Motion 47, 59-65 (2010).

12. Ting, T. C. T.: Existence of anti-plane shear surface waves in anisotropic elastic half-space with depth-dependent material properties. Wave Motion 47, 350-357 (2010).

13. De, S. N., Sengupta, P. R.: Surface waves under the influence of gravity. Gerlands Beitr. Geophys. 85, 311-318 (1976).

14. Dey, S. K., Sengupta, P. R.: Effects of anisotropy on surface waves under the influence of gravity. Acta Geophys. Polonica 24 ZZZ, 291-298 (1978).

15. De, S. N., Sengupta, P. R.: Plane Lamb's problem under the influence of gravity. Gerlands Beitr. Geophys 82, 421-426 (1973).

16 Abd-Alla, A. M., Ahmad, S. M.: Stoneley and Rayleigh waves in a nonhomogeneous orthotropic elastic medium under the influence of gravity. Appl. Math. Computation, 135, 187-200 (2003).

17. Vinh, Pham Chi, Seriani, Geza: Explicit secular equations of Stoneley waves in a non-homogeneous orthotropic elastic medium under the influence of gravity. Appl. Math. Computation, 215, 3515-3525 (2010).

18. Ting, T. C. T.: Surface waves in an exponentially graded general anisotropic elastic material under the influence of gravity. Wave Motion, in print (2011).

19. Stroh, A. N.: Steady state problems in anisotropic elasticity. J. Math. Phys. 4, 77103 (1962).

20. Chadwick, P., Smith, G. D.: Foundations of the theory of surface waves in anisotropic elastic materials. Adv. Appl. Mech. 17, 303-376 (1977). 
21. Ting, T. C. T.: Anisotropic Elasticity: Theory and Applications. Oxford University Press, New York (1996).

22. Biot, M. A.: Mechanics of Incremental Deformation. Wiley, New York (1965).

23. Ingebrigtsen, K. A., Tonning, A.: Elastic surface waves in crystal. Phys Rev. 184, 942-951 (1969).

24. Barnett, D. M., Lothe, J.: Synthesis of the sextic and the integral formalism for dislocations, Greens function and surface waves in anisotropic elastic solids. Phys. Norv. 7, 13-19 (1973).

25. Chadwick, P., Ting, T. C. T.: On the structure and invariance of the Barnett-Lothe tensors. Q. Appl. Math. 45, 419-427 (1987).

26. Barnett, D. M., Chadwick, P., Lothe, J.: The behaviour of elastic surface waves polarized in a plane of materials symmetry. I. Addendum. Proc. Roy. Soc. A433, 699-710 (1991).

27. Chadwick, P.: Some remarks on the existence of one-component surface waves in elastic materials with symmetry. The Jens Lothe Symposium Volume, Physica Scripta T44, 94-97 (1992).

28. Ting, T. C. T.: Surface waves in anisotropic elastic materials for which the matrix $\mathbf{N}(v)$ is extraordinary degenerate, degenerate, or semisimple. Proc. Roy. Soc. London A453, 449-472 (1997).

29. Ting, T. C. T., Barnett, D. M.: Classifications of surface waves in anisotropic elastic materials. Wave Motion 26, 207-218 (1997).

30. Stoneley, R.: Elastic waves at the surface of separation of two solids. Proc Roy. Soc. London A106, 416-428 (1924).

31. Barnett, D. M., Lothe, J., Musgrave, M. J. P., Gavazza, S. D.: Interfacial (Stoneley) waves in bonded anisotropic elastic half-spaces. Proc. Roy. Soc. London A402, 153-166 (1985).

32. K. Sezawa, K. Kanai: The range of possible existence of Stoneley-waves, and some related problems. Bull. Earthq. Res. Inst. Tokyo Univ. 17, 1-8 (1939).

33. P. Chadwick, P. Borejki: Existence and uniqueness of Stoneley waves. Geophys. J. Int. 118, 279-284 (1994). 
34. Ting, T. C. T.: Existence of one-component Rayleigh waves, Stoneley waves, Love waves, slip waves and one-component waves in a plate or a layered plate. The George Herrman Special Issue, J. Mech, Materials and Structures. 4(4), 631-647 (2009). 